\title{
RELACIONES TRÓFICAS ENTRE EQUINOIDEOS (ECHINODERMATA) DE LAS POCETAS INTERMAREALES DE LA SARDINA Y GUAIMARAL, COSTA SURORIENTAL DE CUBA
}

\author{
Asiel Cabrera Guerrero, Rogelio Roberto Muñoz Li, César Daniel Batista Saldívar, \\ Flavia Álvarez Denis y Abdiel Jover Capote ${ }^{1}$
}

Departamento de Biología, Facultad de Ciencias Naturales y Exactas, Universidad de Oriente. Patricio Lumumba s/n, Santiago de Cuba, Cuba, CP: 90500. ajover@uo.edu.cu ${ }^{1}$

\section{RESUMEN}

Se caracterizan las relaciones tróficas de Diadema antillarum Philippi, 1845, Lytechinus variegatus (Lamarck, 1816) y Echinometra viridis A. Agassiz, 1863, mediante la riqueza y diversidad tróficas, amplitud y solapamiento del nicho y sus estrategias de alimentación. Se recolectaron al azar 31 individuos adultos para el análisis del contenido estomacal $(D$. antillarum $=13$, L. variegatus $=13$ y E. viridis $=5)$. En la dieta se identificaron 28 recursos tróficos y el $57 \%$ fueron vegetales. A partir de la amplitud del nicho trófico se concluye que estas especies son consumidores generalistas y su dieta se basa principalmente en recursos tróficos vegetales. Los recursos más abundantes en la dieta fueron las algas filamentosas Polysiphonia y Oscillatoria. Las estrategias de alimentación muestran variaciones interindividuales en el uso de los recursos tróficos y la compartimentación del nicho, lo que presupone la coexistencia sin competencia de sus poblaciones.

Palabras clave: dieta, riqueza trófica, estrategia de alimentación, amplitud del nicho, solapamiento del nicho.

\section{TROPHIC RELATIONSHIPS BETWEEN EQUINOIDS (ECHINODERMATA) OF THE INTERTIDAL TIDE POOLS OF LA SARDINA AND GUAIMARAL, SOUTHEASTERN COAST OF CUBA}

\begin{abstract}
In this work is characterized the relationships of Diadema antillarum Philippi, 1845, Lytechinus variegatus Lamarck, 1816 and Echinometra viridis A. Agassiz, 1863 by the richness and trophic diversity, breadth and overlap of the niche and their feeding strategies. Thirty-one adult individuals were collected at random for analysis of stomach contents $(D$. antillarum $=13$, L. variegatus $=13$ and $E$. viridis $=5)$. In the diet, 28 trophic resources were identified and 57\% were vegetables. From the breadth of the trophic niche these species are general consumers and their diet is mainly based on vegetable trophic resources. The most abundant dietary resources were the Polysiphonia and Oscillatoria filamentous algae. Feeding strategies show interindividual variations in the use of trophic resources and the compartmentalization of the niche, which presupposes the coexistence without competition of their populations.
\end{abstract}

Keywords: diet, trophic richness, feeding strategy, niche breadth, niche overlap. 


\section{INTRODUCCIÓN}

La composición de la dieta determina la coexistencia de las especies en un hábitat y la estructura de las comunidades (Carrete et al., 2010). Por lo que la competencia por un mismo recurso alimenticio constituye un factor limitante en la distribución de los organismos. Además, la existencia de nichos tróficos diferentes es una estrategia desarrollada por organismos sintópicos (Rivas, 1964).

La manera en que se reparte el alimento entre especies que comparten un mismo hábitat es uno de los aspectos esenciales del nicho (Taper y Marquet, 1996). La abundancia en el medio del alimento y las características del hábitat determinan la partición del recurso trófico y son modelados bajo la Teoría del Forrajeo Óptimo (Goszcynski,1986; Clode y MacDonald, 1995). Estos modelos predicen el aumento en la amplitud de nicho con respecto al decremento en la disponibilidad de recursos, pues en estas condiciones se promueve el consumo de un amplio rango de presas subóptimas y la pérdida de especificidad trófica (Svärdson, 1949; MacArthur y Pianka, 1966; Krebs y Davies, 1981).

Entre las especies que coexisten en los hábitats marinos intermareales encontramos a los equinoideos. Estos organismos son importantes en la trama trófica (nicho), pues su dieta está compuesta por algas, angiospermas marinas, corales, moluscos, crustáceos y otros animales (Bronnstein y Loya, 2014). Además, son un grupo taxonómico con elevada capacidad para modificar las condiciones del sustrato en el que viven y controlan la abundancias de los organismos que forman parte de su alimentación (Birkeland, 1989).

En la actualidad, los principales trabajos que se realizan en este grupo taxonómico tienen un carácter descriptivo sobre la relación de los equinoideos y las macroalgas (Bell y Williamson, 2001). También son frecuentes los que abordan su relación con las características del hábitat (Entrambasaguas et al., 2008) y en la estructura del hábitat bentónico (Sala et al., 1998).

Para el Caribe y el Golfo de México se han reportado aproximadamente cien especies de erizos marinos (Hendler et al., 1995). Además, en esta área geográfica los trabajos que aborden la temática de la dieta son pocos (Nappi y Crawford, 1984; Klinger et al., 1986). El enfoque va desde trabajos descriptivos de los componentes de la dieta hasta el empleo de la ecología isotópica (Rodríguez-Barreras et al., 2016). Sin embargo, en hábitats de pocetas intermareales no existen evidencias de si las poblaciones de los equinoideos dominantes coexisten y no compiten entre ellas por los recursos tróficos de su dieta.

\section{OBJETIVO}

-Caracterizar las relaciones tróficas de tres equinoideos dominantes en las pocetas intermareales de Guaimaral y La Sardina, Cuba.

\section{MATERIALES Y MÉTODOS}

Área de estudio. Las tres localidades de estudio están ubicadas cercanas a La Mula, municipio de Guamá, porción sur-occidental de la provincia de Santiago de Cuba. Esta área se caracteriza por poseer una costa alta y acantilada, cortada por la desembocadura de los numerosos ríos que descienden desde la Sierra Maestra. Los sitios corresponden a pocetas intermareales con extensiones superiores a 60 metros cuadrados, protegidas del embate de las olas por una plataforma de rocas de origen volcánico. La costa adyacente es una playa rocosa de cantos rodados con grandes descensos 
y sometida al fuerte oleaje. En el interior de la misma existe gran diversidad de organismos en fondos arenosos y rocosos con formaciones de coral. En Guaimaral se encuentran pequeños parches de la angiosperma marina Thalassia testudinum Banks ex König.

La poceta de La Sardina (fig. 1A) se encuentra ubicada a $2.3 \mathrm{~km}$ de la desembocadura del río Turquino (19.940051 N y $76.776912 \mathrm{~W}$ ) y presenta un litoral rocoso de $6 \mathrm{~m}$ de altura. Su fondo es arenoso con rocas cubiertas con poca vegetación. En tanto, las pocetas del Guaimaral (fig. 1, B y C) se localizan a $1.7 \mathrm{~km}$ de la desembocadura del río Turquino (19.942974 N, $76.771425 \mathrm{~W})$. Su litoral posee una barrera de rocas sedimentarias y volcánicas expuestas al fuerte oleaje con una altura aproximada a $3 \mathrm{~m}$.

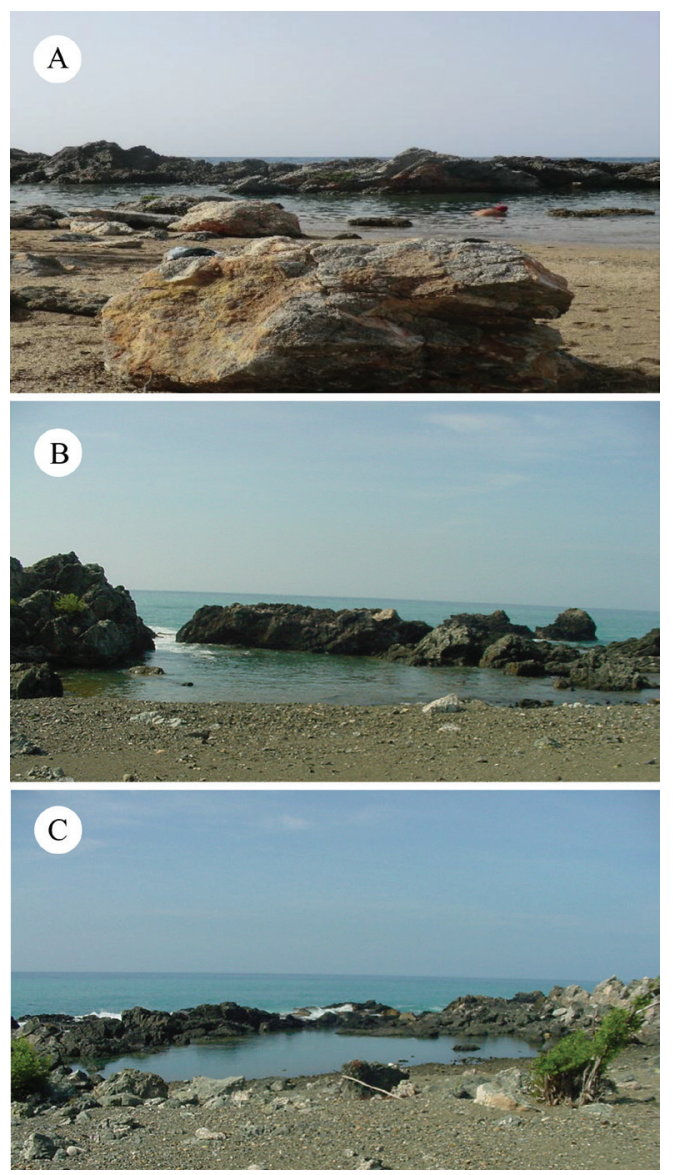

FIGURA 1. Fotografías de las pocetas intermareales de La Sardina y Guaimaral. A, La Sardina. B, Guaimaral II. C, Guaimaral I.

\section{MUESTREO}

Recolección de los individuos. En las localidades de muestreo se seleccionaron las tres especies de equinoideos más abundantes según datos de trabajos previos en el área (Martínez et al., 2016). Para evitar el sesgo de la influencia del tamaño en la alimentación fueron seleccionados ejemplares de tamaño de testa semejante o superior a los $30 \mathrm{~mm}$ de diámetro. A partir de este tamaño los equinoideos son considerados adultos (Montealegre- Quijano y Gómez, 2005). 
El muestreo se realizó al azar y los ejemplares de Diadema antillarum Philippi, Lytechinus variegatus Lamarck y Echinometra viridis A. Agassiz se separaron del sustrato con ayuda de pinzas y se depositaron en contenedores para su posterior procesamiento. Las colectas se realizaron de 7:00-8:30 a. m., de forma tal que todo el contenido estomacal no hubiese sido digerido. Para facilitar las observaciones y mediciones respectivas, se narcotizaron los individuos aplicando formalina al 3\% (Monroy y Solano, 2005).

Análisis del contenido estomacal. Los estómagos fueron sacados con pinzas suaves por medio de una incisión en el poro oral y se depositaron en frascos de vidrio con alcohol al 70\% para su posterior análisis. El contenido estomacal fue analizado bajo un estereoscopio NSZ-808M con una cámara HDCE-50B acoplada, usando el método de conteo de puntos (Jones, 1968) para determinar la abundancia de cada recurso en el estómago (Frantzis et al., 1988). La frecuencia fue usada para determinar la abundancia relativa de cada tipo de alimento en el interior del estómago. El contenido vegetal y animal se identificó con ayuda de un microscopio N-800M con una cámara HDCE-50B acoplada, hasta el nivel taxonómico más bajo posible, mediante la identificación de los caracteres diagnósticos reportados en la literatura especializada y la consulta a especialistas.

\section{ANÁLISIS DE LOS DATOS}

Riqueza trófica. Se estimó como el número de recursos tróficos para las tres especies de equinoideos, usando para ello el modelo de Clench (Ec. 1. Jiménez-Valverde y Hortal, 2003):

$$
S_{n}=\frac{a * n}{1(b * 1)}(\text { Ecuación 1) }
$$

Donde: $\mathrm{S}_{\mathrm{n}}=$ riqueza de especies, $\mathrm{n}=$ número de muestra. La curva de acumulación fue suavizada con 99999 aleatorizaciones en el programa PRIMER versión 6 (Clarke y Gorley, 2006) y el modelo de Clench se construyó en el programa Species Accumulation Functions versión Beta (Díaz-Francés y Soberón, 2005).

Abundancia de los recursos tróficos. Se realizaron curvas de rango-abundancia para el contenido trófico de $D$. antillarum, L. variegatus y E. viridis en base a la contribución de la abundancia relativa de cada recurso presente en la dieta. Estas curvas permiten una comparación gráfica de la riqueza trófica (número de puntos), sus abundancias relativas, la forma de las curvas (equitatividad) y, además, conservan la identidad de las presas que componen el espectro trófico de las especies (Whittaker, 1965).

Amplitud y solapamiento del nicho trófico. La amplitud del nicho es un parámetro que pretende cuantificar qué tan especializada es una especie dentro de un ambiente determinado (Krebs, 1999). Un especialista se alimenta de uno o dos recursos alimenticios y tiene, por lo tanto, una amplitud de nicho menor que el de un generalista. Para esto, se aplicó el índice de Levins (Ec. 2), que cuantifica la uniformidad en los recursos consumidos por cada especie, expresados como:

$$
B=\frac{1}{\Sigma P_{i}^{2}}(\text { Ecuación 2) }
$$

donde $\mathrm{P}_{\mathrm{i}}$ es la proporción de individuos encontrados usando el recurso i. El índice de Levins estandarizado se utilizó para expresar la amplitud de nicho en una escala que va desde 0 (nicho angosto) hasta 1 (nicho amplio). Así, cuando los valores son menores de 0.60 , el organismo se considera especialista, lo que indica que utiliza un número bajo de recursos y presenta una preferencia por ciertos alimentos (Krebs, 1999). 
Para estimar el grado de similitud de la dieta entre las especies, se utilizó un índice que mide el nivel en que dos especies usan un conjunto de recursos en particular y manifiestan el grado de repartición de recursos entre pares de especies, dentro de una comunidad. Se seleccionó el índice de sobreposición de dieta simplificado de Morisita (Ec. 3), expresado como:

$$
\mathrm{C}=\frac{2 \sum_{\mathrm{i}}^{\mathrm{n}} \rho_{\mathrm{ij}} \rho_{\mathrm{ik}}}{\sum_{\mathrm{i}}^{\mathrm{n}} \rho_{\mathrm{ij}}\left[\left(\mathrm{n}_{\mathrm{ij}}\right)^{1} /\left(\mathrm{N}_{\mathrm{j}}-1\right)\right]+\sum_{\mathrm{i}}^{\mathrm{n}} \rho_{\mathrm{ik}}\left[\left(\mathrm{n}_{\mathrm{ik}}-1\right) /\left(\mathrm{N}_{\mathrm{k}}-1\right)\right]}(\text { Ecuación } 3)
$$

Donde Pij es la proporción de un recurso i usado por una especie $\mathrm{j}$, lo mismo para Pik, nij: número de especies que usan un recurso, lo mismo para nik; $\mathrm{Nj}$ y $\mathrm{Nk}$ : numero total de esa especie usados. Este índice varía entre 0 y 1, y se considera una superposición significativa cuando los valores exceden de 0.6 (Langton, 1982). Además, no presenta mayor sensibilidad a la riqueza trófica ni al tamaño de muestra como otros índices (Magurran, 2004). Ambos índices fueron calculados empleando el programa Ecological Methodology versión 6.1.1 (Krebs, 1999).

Estrategia de alimentación. El análisis de la estrategia de alimentación tiene gran importancia en las relaciones entre los componentes de la dieta. Esta última fue analizada usando una modificación del método gráfico de Costello propuesto por Amundsen et al. (1996). En este modelo se realiza una representación bidimensional donde se relacionan la abundancia proporcional de los recursos tróficos y la frecuencia de ocurrencia, dado por la ecuación:

$$
\rho_{\mathrm{i}}=\frac{\left(\sum \mathrm{s}_{\mathrm{i}}\right)}{\left(\sum \mathrm{s}_{\mathrm{ti}}\right)} * 100(\text { Ecuación } 4)
$$

Donde: $\mathrm{S}_{\mathrm{i}}$ es el número de contenido estomacal conformado por cada presa y $\mathrm{S}_{\mathrm{ti}}$ el total de estómagos que contienen un recurso determinado. Para la interpretación del diagrama los dos ejes diagonales representan la importancia del recurso trófico (dominante o raro) y la contribución a la amplitud del nicho (AANTI-alta amplitud del nicho trófico individual y AANTP alta amplitud del nicho tófico poblacional) y el eje vertical define la estrategia alimentaria del depredador (especialista y generalista; fig. 2).

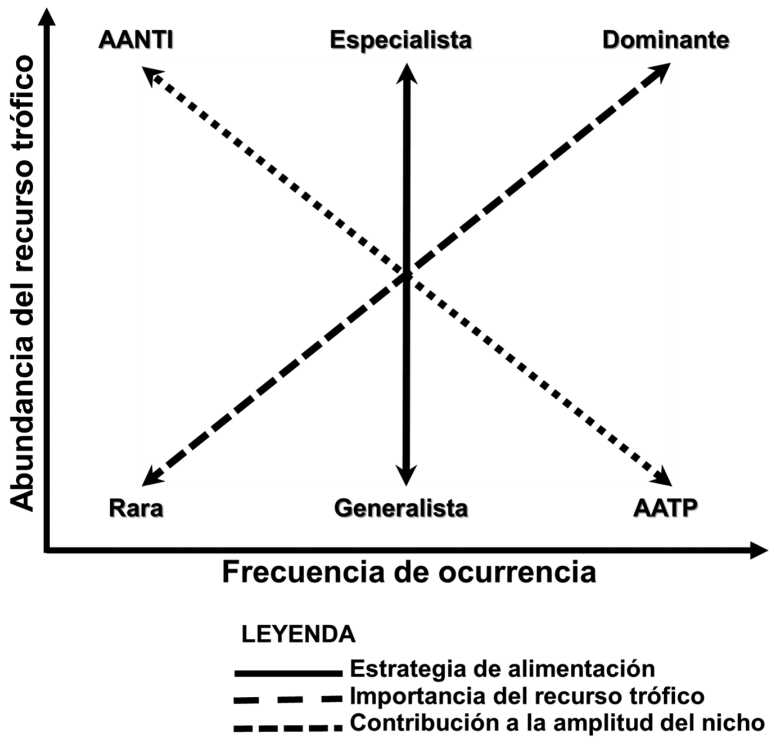

FIGURA 2. Diagrama explicativo (centro) para la interpretación de la estrategia de alimentación, la contribución a la amplitud del nicho (AANTI-alta amplitud del nicho trófico individual y AANTP-alta amplitud del nicho trófico poblacional) y la importancia del recurso trófico en las líneas diagonales (modificado de Amundsen et al., 1996). 


\section{RESULTADOS}

Composición y riqueza de recursos tróficos de la dieta. En la dieta de los equinoideos dominantes de las pocetas intermareales de Guaimaral y La Sardina se identificó un total de 28 recursos tróficos. El 57\% fueron vegetales, de los cuales el 17\% constituyen algas verdes, $35 \%$ algas rojas, el $2 \%$ algas pardas y el $3 \%$ espermatofitas. Los alimentos de origen animal fueron los menos frecuentes pues se observaron solo representantes de cinco phyla. Los elementos que se identificaron fueron fragmentos de esponjas, corales, anélidos, hidrozoos, moluscos y crustáceos; lo que representa el $32 \%$ de los recursos identificados (Tabla I).

La dieta de $D$. antillarum estuvo constituida por 26 de los 28 recursos tróficos identificados en total $(92.857 \%$ ) y es la especie de mayor riqueza trófica. Se identificaron cinco morfoespecies de algas verdes, nueve de algas rojas, una de alga parda, un taxon de cianobacterias y otro de angiospermas. Además, se observaron siete grupos de animales y uno de protozoarios. Esta especie fue la única que presentó entre los componentes de su dieta fragmentos de Caulerpa, así como de pequeños bivalvios y malacostracos.

En L. variegatus se identificaron 24 de los 28 recursos tróficos identificados $(85.71 \%)$ en los estómagos diseccionados. La mayor riqueza trófica, al igual que en D. antillarum, lo constituyeron los recursos vegetales, identificándose cuatro clorofíceas, ocho rodofíceas y un taxa de feofícea, uno de diatomea y otro de angiosperma. Además se identificaron como parte de su alimentación seis phyla de animales y uno de protozoos. En esta especie de equinoideo el recurso trófico exclusivo fue Acanthophora (alga roja).

Por último, la especie con menor riqueza trófica fue E. virides con 11 recursos identificados (39.29\%). El único alimento animal presente en su alimentación fueron fragmentos de corales. En tanto, el resto estuvo compuesto por cinco algas rojas y un recurso perteneciente a los phyla de Chlorophyta, Cyanobacteria y Phaeophyta.

Las curvas de acumulación de especies, muestran que el muestreo realizado no fue suficiente para determinar la riqueza trófica estimada (fig. 3). El número tentativo de ejemplares que deberían ser recolectados tendría que estar cerca de 45 para $D$. antillarum, 35 para $L$. variegatus y 34 para E. viridis, para reducir el sesgo del tamaño de muestra de las estimaciones.

En $D$. antillarum se identificó el $87 \%$ de los recursos tróficos estimados (Ecuación de Clench: $\mathrm{S}_{\mathrm{obs}}=26 ; \mathrm{R}^{2}=0.9998 ; \mathrm{a} / \mathrm{b}=29.78 ;$ pendiente $=0.29$ ). Por su parte, en L. variegatus se identificó el $86 \%$ de los alimentos de su dieta (Ecuación de Clench: $\mathrm{S}_{\mathrm{obs}}=24 ; \mathrm{R}^{2}=0.9968 ; \mathrm{a} / \mathrm{b}=27.98$; pendiente $=0.23$ ). Estas dos especies mostraron una tendencia a alcanzar la asíntota, lo que sugiere que el tamaño de muestra seleccionado se acerca al estimado para describir la dieta. En el caso de E. viridis el tamaño de muestra es más limitado para describir los elementos de su dieta, aunque se determinó el 76\% del estimado (Ecuación de Clench: $\mathrm{S}_{\mathrm{obs}}=11 ; \mathrm{R}^{2}=0.9887 ; \mathrm{a} / \mathrm{b}=14.53$; pendiente $=0.56$ ).

Abundancia de recursos tróficos. Las curvas de rango-abundancia representadas para las tres especies de equinoideos de las pocetas intermareales de La Sardina y Guaimaral mostraron un patrón de distribución de abundancia equitativa (fig. 4). Las especies que mostraron patrones de distribución de la abundancia de sus recursos tróficos más equitativas fueron $D$. antillarum y $L$. variegatus, mientras que en $E$. viridis la distribución de su abundancia fue poco equitativa. 
TABLA I.

Recursos tróficos presentes en la dieta de los equinoideos dominantes en las pocetas intermareales de La Sardina y Guaimaral. Donde: A-La Sardina, B-Guaimaral I y C-Guaimaral II.

\begin{tabular}{|c|c|c|c|c|c|c|c|}
\hline \multirow{2}{*}{ Recursos Tróficos } & \multicolumn{3}{|c|}{ D. antillarum } & \multicolumn{3}{|c|}{ L. variegatus } & \multirow{2}{*}{$\frac{\text { E. viridIs }}{\mathrm{C}}$} \\
\hline & A & $\mathrm{B}$ & $\mathrm{C}$ & $\mathrm{A}$ & $\mathrm{B}$ & $\mathrm{C}$ & \\
\hline \multicolumn{8}{|l|}{ Cyanobacteria } \\
\hline Oscillatoria & 13 & 21 & 24 & 25 & 47 & - & 2 \\
\hline \multicolumn{8}{|l|}{ Rodophyta } \\
\hline Galaxaura & 1 & - & - & 7 & - & - & - \\
\hline Ceramium & 5 & 1 & - & 3 & - & - & 3 \\
\hline Polysiphonia & 19 & 21 & 2 & 6 & 37 & - & 82 \\
\hline Cladophora & 12 & 22 & 14 & 17 & 3 & - & 1 \\
\hline Chaetomorpha & 1 & 2 & - & 5 & 12 & - & - \\
\hline Laurencia & 1 & - & - & 2 & - & - & - \\
\hline Amphiroa & 2 & 19 & - & 12 & - & - & 1 \\
\hline Gracilaria & 25 & 16 & 12 & 19 & 53 & 4 & 1 \\
\hline Dasya & - & 21 & - & - & - & - & - \\
\hline Acanthophora & - & - & - & - & 2 & - & - \\
\hline Jania & - & - & 4 & - & 5 & - & 55 \\
\hline Centrocera & 8 & 16 & - & 13 & 9 & 13 & - \\
\hline \multicolumn{8}{|l|}{ Clorophyta } \\
\hline Ulva & 5 & 3 & - & 2 & - & - & - \\
\hline Caulerpa & - & 1 & - & - & - & - & - \\
\hline Valonia & 1 & - & - & 1 & 32 & - & - \\
\hline \multicolumn{8}{|l|}{ Pheophyta } \\
\hline Dictyota & 3 & - & 4 & 5 & 7 & - & 19 \\
\hline Bacillariophyta & 7 & 5 & - & 2 & 4 & - & 7 \\
\hline \multicolumn{8}{|l|}{ Spermatophytas } \\
\hline Thalassia & - & - & 23 & - & 32 & - & - \\
\hline Foraminifera & 6 & 6 & - & 7 & 1 & - & 2 \\
\hline Porifera & 4 & - & 3 & - & 7 & - & - \\
\hline \multicolumn{8}{|l|}{ Coelenterata } \\
\hline Anthozoa & 8 & 2 & 16 & 23 & - & - & 7 \\
\hline Hydrozoa & - & - & - & 1 & - & - & - \\
\hline Annelida & 1 & 2 & - & 2 & - & - & - \\
\hline \multicolumn{8}{|l|}{ Mollusca } \\
\hline Bivalvia & 1 & - & - & - & - & - & - \\
\hline Gasteropoda & 5 & 4 & 3 & 4 & 1 & - & - \\
\hline \multicolumn{8}{|l|}{ Crustacea } \\
\hline Ostracoda & 3 & 1 & 1 & 2 & - & - & - \\
\hline Malacostraca & - & - & 1 & - & - & - & - \\
\hline
\end{tabular}

-Ausencia del recurso. 
Los recursos tróficos más abundantes en $D$. antillarum fueron grupos de algas pertenecientes a los géneros Polysiphonia, Oscillatoria y Gracilaria, aportando el 36\% de la abundancia total (fig. 4). Luego se ubican 17 recursos de abundancia intermedia en donde aparecen nueve géneros de algas, cinco de animales y uno de angiosperma. En el extremo inferior se ubican los recursos menos abundantes en la dieta de esta especie que son cuatro algas (Caulerpa, Galaxaura, Valonia y Laurencia) y dos animales (Bivalvia y Malacostraca).

En la dieta de L. variegatus las algas Gracilaria y Oscillatoria aportan el 31\% de la abundancia total (fig. 4). En esta especie se consigna el mayor número de especies con abundancia intermedia 13 algas, seis animales y una angiosperma. Además, es en donde solo se reporta un recurso en el extremo de la curva que es Hidrozoa.

El alga Polysiphonia, como recurso dominante en la dieta de E. viridis, aportó el 37\% de la abundancia. En esta especie se reporta el menor número de elementos en los estómagos identificándose seis algas y dos animales. En el extremo de la curva se ubican los géneros Cladophora y Amphiroa, que son los recursos menos abundantes.

Amplitud y solapamiento del nicho trófico. Los mayores valores de amplitud del nicho trófico de Levin lo mostraron $D$. antillarum con $\mathrm{B}=0.415$ y L. variegatus con $\mathrm{B}=0.423$ (Tabla II), mientras que $E$. viridis, con una amplitud de $\mathrm{B}=0.318$, resultó la especie de menor amplitud de nicho. El índice simplificado de Morisita mostró que entre D. antillarum y L. variegatus existe un solapamiento muy importante de sus dietas $(\mathrm{C}=0.92)$. El solapamiento del nicho de E. viridis con las otras dos especies no se considera importante, pues posee valores inferiores a 0.6. La amplitud del nicho de D. antillarum y L. variegatus en las pocetas intermareales de La Sardina y Guaimaral I muestran valores semejantes al comportamiento de estas especies para la localidad (Tabla II). En la primera localidad la mayor amplitud la posee $L$. variegatus $(\mathrm{B}=0.49)$ y en la segunda localidad lo posee D. antillarum $(\mathrm{B}=0.54)$. Además, entre estas dos especies al parecer existe un fuerte solapamiento de sus recursos tróficos en ambas localidades con valores del índice de Morisita superiores a 0.7.

En la localidad de Guaimaral II no se evidencia un importante solapamiento del nicho trófico de D. antillarum con L. variegatus y E. viridis (Tabla II). Tampoco se muestran evidencias de competencia por los recursos tróficos entre L. variegatus y E. viridis pues el valor del índice de solapamiento entre ellas es el más bajo $(\mathrm{C}=0.09)$.

Estrategia de alimentación. El análisis gráfico de la estrategia de alimentación de los equinoideos $D$. antillarum, $L$. variegatus y $E$. viridis muestra un comportamiento semejante en la distribución de los alimentos encontrados en su dieta (fig. 5). En estos equinoideos $>50 \%$ de los recursos tróficos se ubican en la parte inferior de la figura, por lo que se pueden catalogar como especies generalistas con algunas diferencias entre ellas.

En las poblaciones de $D$. antillarum se observa cómo sus individuos contribuyen de manera diferencial a la amplitud del nicho de la especie (fig. 5A). El 30\% de los individuos se alimentan de las especies más abundantes en la dieta como son la cianobacteria Oscillatoria y la rodofícea Polysiphonia. Sin embargo, más del $70 \%$ de los individuos tienen como componentes en su alimentación recursos que no son abundantes como foraminíferos, anélidos y las algas Jania, Valonia y Chaetomorpha. No se identificó ningún recurso como dominante en la dieta de esta especie y el 57\% constituyen elementos raros. 
A diferencia del comportamiento de $D$. antillarum en las poblaciones de L. variegatus más del $50 \%$ de sus individuos no consumen ningún recurso trófico en abundancia (fig. 5B). Además, para esta especie la macroalga Gracilaria es dominante en la dieta de más del $60 \%$ de sus individuos. Otros alimentos consumidos por más del $50 \%$ de la población son Oscillatoria, Cladophora y Polysiphonia. El 54\% de los recursos tróficos aparecen como raros en la dieta, pues son consumidos por menos del $50 \%$ de la población en pocas cantidades.
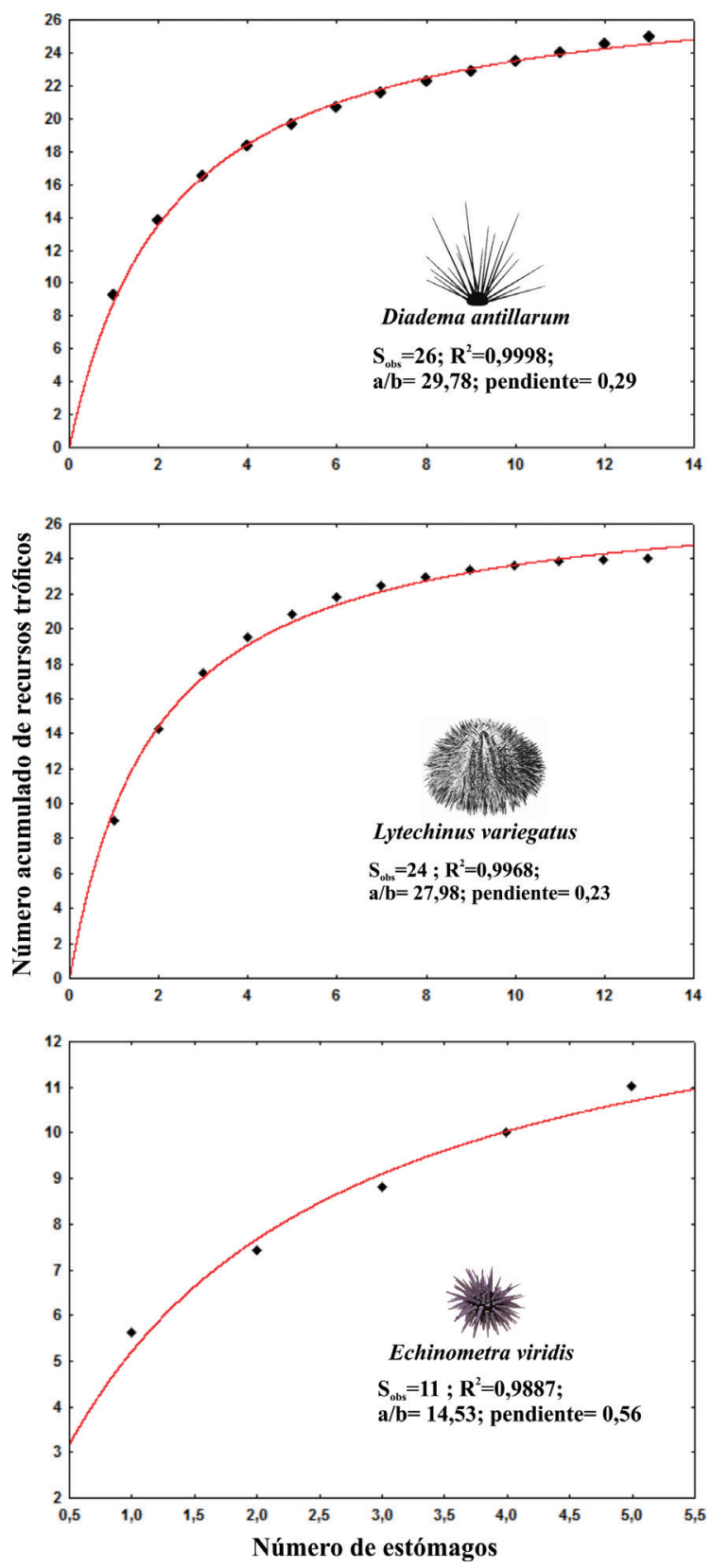

FIGURA 3. Curvas de acumulación de especies para los equinoideos dominantes de las pocetas intermareales de La Sardina y Guaimaral. 
Los individuos de la población de $E$. viridis también contribuyen de manera diferencial a la amplitud del nicho trófico de la especie (fig. 5C). Esto se explica porque en el 20\% de los mismos se observa la abundancia de fragmentos de corales y del alga coralina Jania. El 80\% de la población consume con abundancia el alga Polysiphonia, siendo este recurso dominante en su dieta. Además, toda la población se alimenta de otros recursos tróficos pocos abundantes como son diatomeas, Ceramium y Gracilaria. El 45\% de los elementos encontrados en los estómagos de esta especie se consumieron por menos del $40 \%$ de la población.

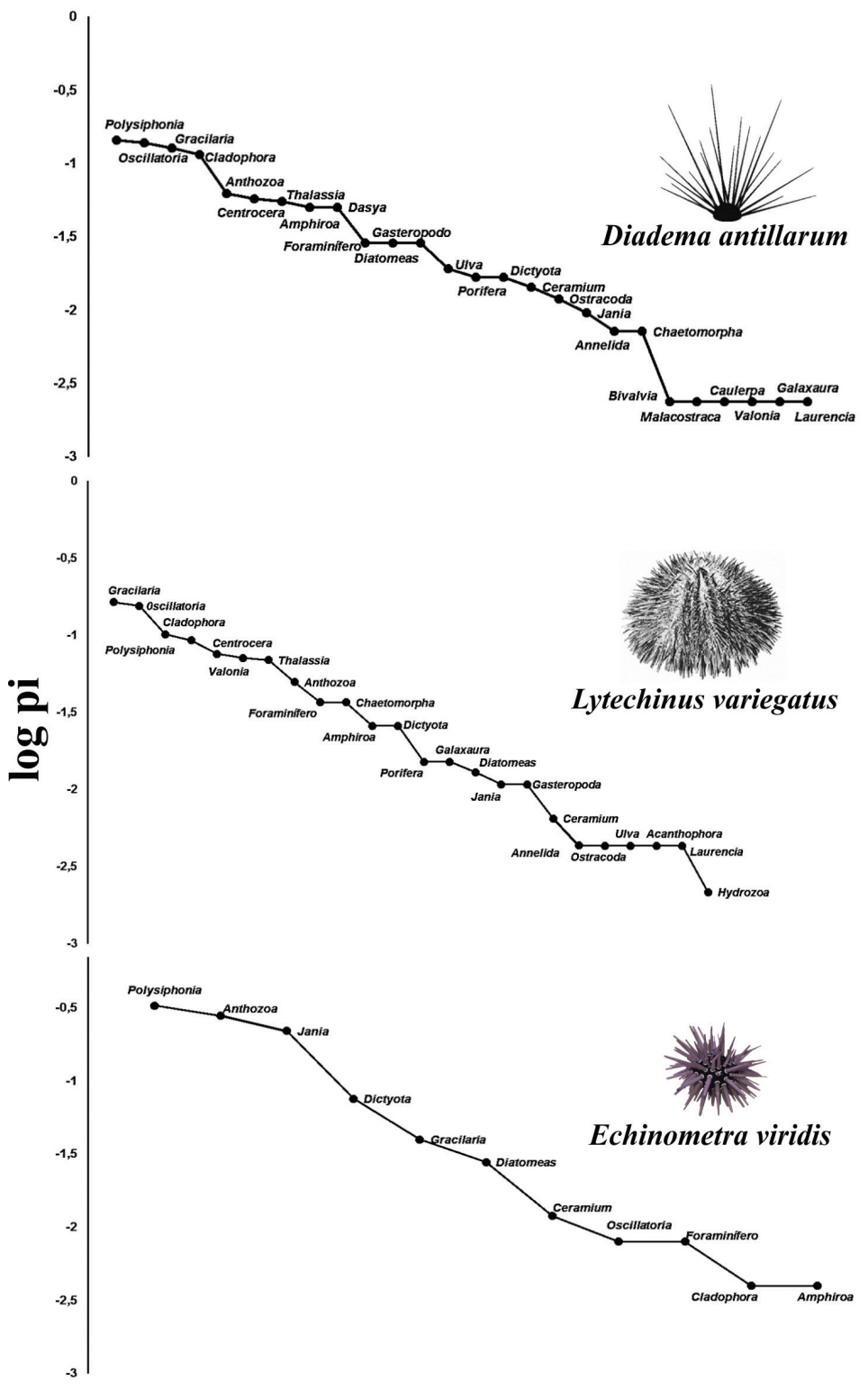

FIGURA 4. Curva de rango-abundancia de los recursos tróficos presentes en la dieta de los equinoideos dominantes en las pocetas intermareales de La Sardina y Guaimaral. 


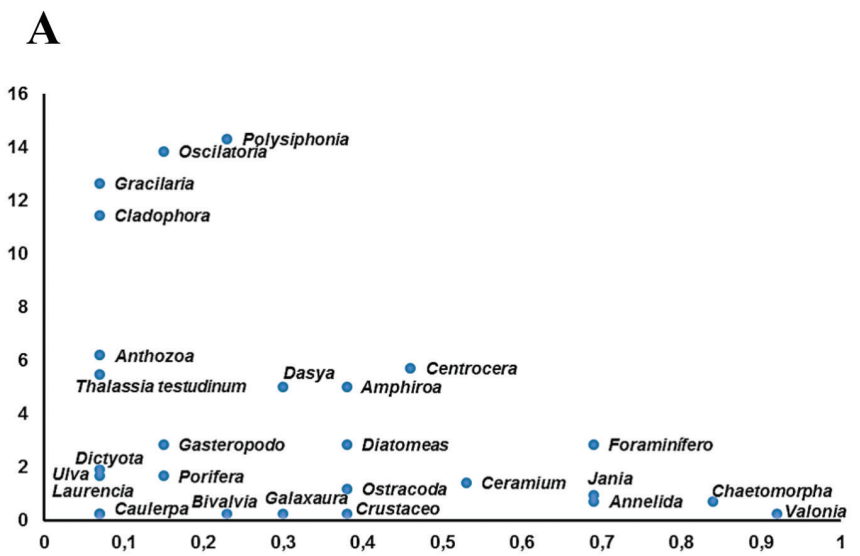

\section{B}

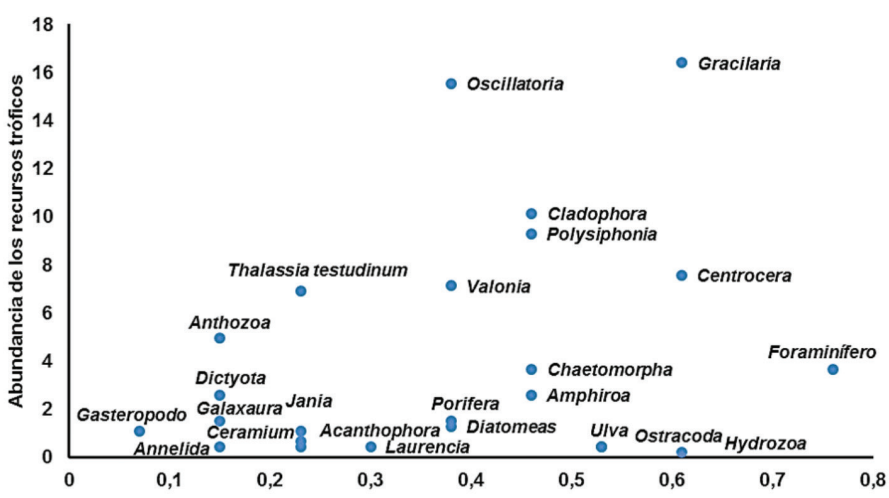

C

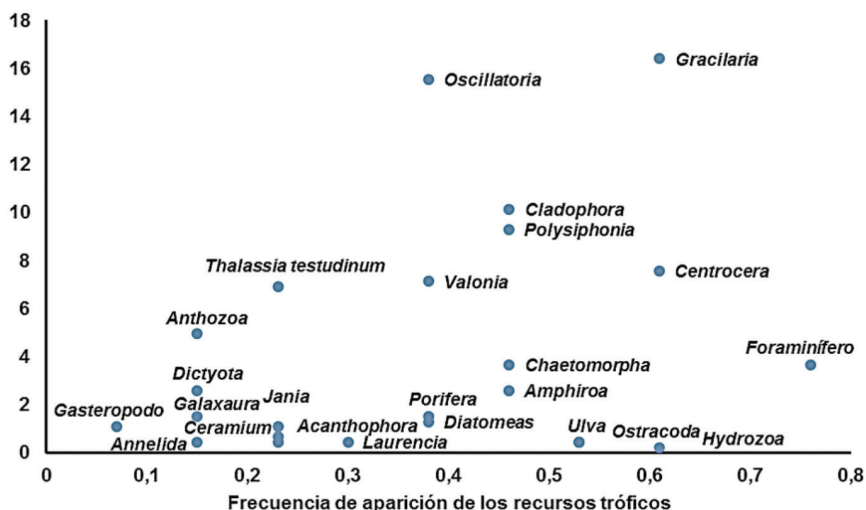

FIGURA 5. A-C. Estrategia de alimentación e importancia de los recursos tróficos en la dieta de los tres equinoideos dominantes de las pocetas intermareales de La Sardina y Guaimaral. A, Diadema antillarum. B, Lytechinus variegatus. C, Echinometra viridis. 


\section{TABLA II.}

Índice de amplitud del nicho trófico (B) y solapamiento trófico (C) de los equinoideos dominantes de las pocetas intermareales de La Sardina y Guaimaral. Donde D. ant. = Diadema antillarum, L. var. = Lytechinus variegatus y E. vir. = Echinometra viridis.

\begin{tabular}{|c|c|}
\hline (A) La Sardina & (B) Guaimaral I \\
\hline $\begin{array}{c}\mathrm{B}(\text { D. ant } .)=0.47 \\
\mathrm{C}(\text { D. ant. X L. var. })=0.8\end{array}$ & $\begin{array}{cc}\mathrm{B}(\text { D. ant } .)=0.54 & \mathrm{~B}(L . \text { var. })=0.5 \\
\mathrm{C}(D . \text { ant. } \mathrm{X} L \text {. var. })=0.71\end{array}$ \\
\hline (C) Guaimaral II & (D) General \\
\hline $\mathrm{B}($ L. var. $)=0.49$ & $\mathrm{~B}($ D. ant $)=0.415$ \\
\hline $\mathrm{B}($ E. vir. $)=0,32$ & $\mathrm{~B}($ E. vir. $)=0,318$ \\
\hline $\mathrm{C}(D$. ant. $\mathrm{X} L$. var. $)=0.4$ & $\mathrm{C}(D$. ant. $\mathrm{X} L$. var. $)=0.92$ \\
\hline $\mathrm{C}($ D. ant. $\mathrm{X} E$. vir. $)=0.55$ & $\mathrm{C}($ D. ant. X E. vir. $)=0.46$ \\
\hline $\mathrm{C}($ L. var. $\mathrm{X}$ E. vir. $)=0.09$ & $\mathrm{C}($ L.var. $\mathrm{X}$ E. vir. $)=0.35$ \\
\hline
\end{tabular}

\section{DISCUSIÓN}

El espectro trófico obtenido para D. antillarum, L. variegatus y E. virides en las pocetas intermareales de La Sardina se corresponde con el descrito para estas especies (Muthiga y McClanahan, 2007; McClanahan y Muthiga, 2013; Wattsa et al., 2007). Según estos autores, estos equinoideos se caracterizan por ser omnívoros. Las diferencias de riqueza trófica de E. virides, comparada con las dos especies restantes, pudieran estar condicionadas por el tamaño de muestra. Sin embargo, para las tres especies, se identificaron más de $75 \%$ de los recursos tróficos y proporciones superiores al $70 \%$ de las estimaciones se consideran como resultados confiables (Jiménez-Valverde y Hortal, 2003).

La mayor riqueza trófica y el patrón de abundancia equitativo observado en $D$. antillarum está dado porque esta especie se alimenta mediante el pastoreo de las algas y del detritus (Muthiga y McClanahan, 2007). Además, Williams (1980) y Shulman (1990), coinciden que en el Caribe $D$. antillarum es un competidor por los recursos porque muestra una actividad mas intensa y agresiva que otras especies estudiadas.

La gran diversidad de algas filamentosas como Polysiphonia y Oscillatoria se debe a la gran cantidad de algas formadoras de césped que ingieren junto al sustrato durante su alimentación (Lewis, 1964). El patrón de distribución de los recursos tróficos en las curvas de rango-abundancia dominado por las algas y en menor proporción por animales se debe a las características del hábitat. Se han encontrado en el tracto digestivo de especies del género Diadema: $28 \%$ de algas, $20 \%$ de angiospermas marinas y $2 \%$ de invertebrados (McClanahan, 1988). Las algas presentes en el extremo inferor de la curva son consideradas como no atractivas en la dieta, caso de Galaxaura (Muthiga y McClanahan, 2007) o producen defensas químicas contra la herbivoría, casos de Caulerpa y Valonia (Hay y Paul, 1986).

El patrón descrito de riqueza y abundancia trófica de L. variegatus se debe a su estrategia de especie omnívora, semejante al de D. antillarum (Wattsa et al., 2007). En la dieta de poblaciones de este equinoideo en las costas de La Florida (Cobb y Lawrence, 2005) y Brasil (Ferreira et al., 2008) se han encontrado gran variedad de vegetales e invertebrados. La abundancia de algas de pequeño tamaño se debe a la preferencia por consumir las epífitas que crecen sobre macroalgas y angiospermas (Greenway, 1995). Además, en hábitats donde estos recursos son abundantes L. variegatus los consume en grandes cantidades (Cobb y Lawrence, 2005). También esta especie es sensible a la presencia de metabolitos secundarios como los presentes en los géneros de algas Caulerpa, Valonia y Laurencia, que son especies de poca abundancia existentes en la dieta (Hay y Fenical, 1988). 
De las tres especies estudiadas es $E$. virides la que posee las características típicas de una especie herbívora generalista (McClanahan y Muthiga, 2013). Según estos autores la base alimentaria de las especies del genéro Echinometra radica en las macroalgas filamentosas formadoras de céspedes, las cianobacterias y las algas que crecen asociadas a los corales. Sin embargo de todos los equinoideos esta es la especie en la que menos animales aparecen en su dieta (McClintock et al., 1982). Los pocos recursos tróficos animales presentes en los estómagos, corales y esponjas fundamentalmente pueden ser ingeridos asociado a la ingestión de las algas. La presencia de pequeños bivalvos, gasterópodos y foraminíferos como recursos de abundancia baja e intermedia en la alimentación de D. antillarum y L. variegatus puede deberse al gran volumen de detritus que ingieren (Beddingfield, 2000; Watts et al., 2007). También estos organismos o fragmentos de otros pudieran estar asociados a los recursos frecuentes en la dieta de estos equinoideos. Además, otros recursos tróficos reportados por su contribución de la dieta de L. variegatus son animales bentónicos como los moluscos, crustáceos y otros pequeños epibiontes (Watts et al., 2007).

La presencia de pequeños invertebrados en la dieta de D. antillarum, L. variegatus y $E$. virides no presupone que persigan, ataquen o capturen activamente estas presas, sino que su consumo sea incidental (Cobb y Lawrence, 2005). Esto explica la baja abundancia en la dieta de muchos de estos recursos. Este fenómeno ha sido descrito para otros herbívoros generalistas de hábitats intermareales (Camus et al., 2013) Aunque no se descarta el aporte que pudiera brindar como fuente de nitrógeno (Reyes-Lujan et al., 2015) y carbonato de calcio (Rodríquez-Barreras et al., 2015).

Los valores intermedios de amplitud del nicho trófico observada en $D$. antillarum, L. variegatus y E. virides evidencian la condición de especies generalistas polífagas, pues consumen recursos pertenecientes a más de un nivel trófico (Pimm y Lawton, 1978). Es probable que las diferencias de amplitud del nicho trófico existente entre las localidades sea por la riqueza de recursos existentes (Bell y Williamson, 2017). En tanto, las diferencias entre las especies responden a características intrínsecas (tipos de enzimas digestivas, estado nutricional y estado reproductivo) al nicho específico como estrategias para optimizar la amplitud del nicho.

El solapamiento trófico de especies coexistentes puede ocasionar competencia en la utilización del recurso alimenticio. Sin embargo, entre los equinoideos estudiados no parece existir competencia por los valores de riqueza trófica. Entre estas especies al parecer, se aprecia una especialización trófica y una repartición diferencial de recursos como se muestran en los diagramas de estrategias de alimentación (Vanderklift et al., 2006). Estos fenómenos se observan en especies sintópicas de equinoideos Además, la coexistencia sin competencia entre las especies estudiadas puede estar relacionada con la plasticidad trófica de los equinoideos (Pinnegar y Polunin, 2000; Wing et al., 2008) lo que permite explotar diferentes fuentes de alimentos. Las estrategias de alimentación de D. antillarum, L. variegatus y E. viridis confirma el carácter generalista de la dieta y la presumible competencia entre estas especies sintópicas. Los aportes a la amplitud del nicho individual presentes en $D$. antillarum y E. virides es característico de otros consumidores del intermareal rocoso (Camus et al., 2013) y responde a la diversidad individual (Bolnick et al., 2002). En estas especies se manifiestan variaciones en el ancho de nicho en la población y en los niveles individuales pues se desacoplan y siguen patrones diferentes de explotación de los recursos (Bolnick et al., 2007, 2010).

En el caso de D. antillarum y L. variegatus las estrategias de alimentación de sus dietas confirman la ausencia de competencia a pesar de los elevados valores de solapamiento del nicho $(>0.6)$ por la compartimentación de sus poblaciones en el hábitat. 
D. antillarum es típica y vive en fondos rocosos y L. variegatus en fondos arenosos asociados a vegetación marina (Rodríguez-Barreras et al., 2016). Estos autores encontraron que entre estas especies no existen solapamientos del nicho como resultado de la compartimentación de los recursos. Estos resultados reafirman el bajo fundamento estadístico para establecer la significación de los estimadores de superposición del nicho trófico (Cailliet y Barry, 1979).

En las pocetas intermareales de La Sardina y Guaimaral las relaciones tróficas que se establecen entre los equinoideos $D$. antillarum, $L$. variegatus y $E$. viridis poseen dietas muy similares a nivel de especie y sus recursos tróficos más frecuentes tienden a ser los mismos sin evidencias de competencia interespecífica. Las especializaciones individuales, en las poblaciones de estos equinoideos, podrían ser efectiva para reducir la competencia intra e interespecífica (Aguilera y Navarrete, 2012). Estas variaciones interindividuales en el uso de los recursos tróficos pueden estar relacionadas con las características del animal (Dall et al., 2012).

\section{CONCLUSIONES}

Los equinoideos Diadema antillarum, Lytechinus variegatus y Echinometra viridis en las pocetas intermareales de La Sardina y Guaimaral son consumidores generalistas, cuya dieta se basa principalmente por recursos tróficos vegetales. Los recursos tróficos más abundantes en la dieta fueron las algas filamentosas Polysiphonia y Oscillatoria. Las estrategias de alimentación de los equinoideos $D$. antillarum, L. variegatus y E. viridis en las pocetas intermareales de La Sardina y Guaimaral muestran variaciones interindividuales en el uso de los recursos tróficos y la compartimentación del nicho lo que presupone la coexistencia sin competencia de sus poblaciones.

\section{AGRADECIMIENTOS}

Se agradece Facultad de Ciencias Naturales por los recursos logísticos que permitieron la realización de la presente investigación. A Yander Luis Diez García por su apoyo en la identificación de los invertebrados. A Luis Ángel Texidor Castellanos por su contribución en la colecta y procesamiento de algunos ejemplares. En especial a Bernardo Reyes Tur por las sugerencias que ayudaron a mejorar el manuscrito y a Alejandro Catalá por el procesamiento de las figuras.

\section{LITERATURA CITADA}

Aguilera M. A. y S. A. Navarrete. 2012. Interspecific competition for shelters in territorial and gregarious intertidal grazers: Consequences for individual behaviour. PLoS ONE, 7: e46205.

Amundsen, P. A., H. M. Gabler y F. J. Staldvik. 1996. A new approach to graphical analysis of feeding strategy from stomach contents data-modification of the Costello (1990) method. Journal of Fish Biology, 48 (4): 607-614.

Beddingfield, S. D. y J. B. McClintock. 2000. Demographic characteristics of Lytechinus variegatus (Echinoidea: Echinodermata) from three habitats in a North Florida Bay, Gulf of Mexico. Marine Ecology, 21 (1): 17-40.

Bell, J. E. y J. E. Williamson. 2017. Positive Indirect Interactions in Marine Herbivores and Algae. Herbivores, 1: 135-153. 
Birkeland, C. h. 1989. The influence of echinoderms on coral reef communities, p. 1-79. En: Echinoderm Studies. M. Jangoux y J. M. Lawrence (eds.). Balkema, Rotterdam, Brookfield, Estados Unidos de America, 382 pp.

Bolnick, D. I., R. Svanbäck, J. A. Fordyce, L. H. Yang, J. M. Davis, C. D. Hulsey y M. L. Forister. 2002. The ecology of individuals: incidence and implications of individual specialization. The American Naturalist, 161 (1): 1-28.

Bolnick D. I., R. Svanbäck, M. S. Araújo y L. Persson. 2007. Comparative support for the niche variation hypothesis that more generalized populations also are more heterogeneous. Proceedings of the National Academy of Sciences of the United States of America, 104: 10075-10079.

Bolnick D. I., T. Ingram, W. E. Stutz, L. K. Snowberg, O. L. Lau y J. S. Paull. 2010. Ecological release from interspecific competition leads to decoupled changes in population and individual niche width. Proceedings of the Royal Society B, 277: 1789-1797.

Bronstein, O. y L. Loya. 2014. Echinoid community structure and rates of herbivory and bioerosion on exposed and sheltered reefs. Journal of Experimental Marine Biology and Ecology, 456: 8-17.

Cailliet, G. M. y J. P. Barry. 1979. Comparison of food array overlap measures useful in fish feeding habit analysis. Gutshop, 78: 67-79.

Camus, P. A., P. A. Arancibia y I. Ávila-Thieme. 2013. A trophic characterization of intertidal consumers on Chilean rocky shores. Revista de Biología Marina y Oceanografía, 48 (3): 431-450.

Carrete M, S. A. Lambertucci, K. Speziale, O. Ceballos, A. Travaini, M. Delibes, F. Hiraldo y J. A. Donázar. 2010. Winners and losers in human-made habitats: interspecific competition outcomes in two Neotropical vultures. Animal Conservation, 13: 390-398.

Clode, D. y D. W. MacDonald. 1995. Evidence for food composition between mink (Mustela vision) and otter (Lutra lutra) on Scottish island. Journal of Zoology London, 237: 435-444.

Cobb, J. y J. Lawrence. 2005. Diets and coexistence of the sea urchins Lytechinus variegatus and Arbacia punctulata (Echinodermata) along the central Florida gulf coast. Marine Ecology Progress Series, 295: 171-182.

Dall S. R. X., A. M. Bell, D. I. Bolnick y F. L. W. Ratnieks. 2012. An evolutionary ecology of individual differences. Ecology Letters, 15: 1189-1198.

Díaz-Francés, E. y J. Soberón. 2005. Statistical estimation and model selection of speciesaccumulation functions. Conservation Biology, 19 (2): 569-573.

Entrambasaguas, L., Á. Pérez-Ruzafa, J. A. García-Charton, B. Stobart y J. J. Bacallado. 2008. Abundance, spatial distribution and habitat relationships of echinoderms in the Cabo Verde Archipelago (eastern Atlantic). Marine and Freshwater Research, 59 (6): 477-488. 
Ferreira, C., A. Santos de Oliveira y R. Crespo. 2008. Feeding preference of the sea urchin Lytechinus variegatus (Lamarck, 1816) on seaweeds. Brazilian Journal of Oceanography, 56: 239-247.

Frantzis, A., J. F. Berthon y F. Maggiore. 1988. Relations trophiques entre les oursins Arbacia lixula et Paracentrotus lividus (Echinoidea Regularia) et le phytobenthos infralittoral superficiel dans la baie de Port-Cros (Var, France). Scientific Reports of Port-Cros national Park, 14: 81-140.

Goszcynski, J. 1986. Diet of foxes and martens in central Poland. Acta Theriologica, 31: 491-506.

Greenway, M. 1995. Trophic relationships of macarofauna within a Jamaican seagrass meadow and the role of the echinoid Lytechinus variegatus (Lamarck). Bulletin of Marine Science, 56: 719-736.

Hay M. E. y W. Fenical. 1988. Marine plant-herbivore interactions: The ecology of chemical defense. Annual Review of Ecology and Systematics, 19:111-145.

Hay M. E. y V. J. Paul. 1986. Seaweed susceptibility to herbivory: chemical and morphological orrelates. Marine Ecology Progress Series, 33: 255-264.

Hendler, G., J. E. Miller, D. L. Pewson y P. M. Kier. 1995. Sea star, Sea urchin and Allies: Echinoderms of Florida and the Caribbean. Smith. Inst. Washington, 390 pp.

Jiménez-Valverde, A. y J. Hortal. 2003. Las curvas de acumulación de especies y la necesidad de evaluar la calidad de los inventarios biológicos. Revista Ibérica de Aracnología, 8 (31): 151-161.

Jones, R. S. 1968. A suggested method for quantifying gut contents in herbivorous fishes. Micronesica, 4 (2): 369-371.

Klinger, T., S. Sieh, R. Pangallo, C. Chen, y J. Lawrence. 1986. The effect of temperature on feeding, digestion and absorption of Lytechinus variegatus (Lamarck). Physiological Zoology, 59 (3): 332-336.

Krebs, C. J. 1999. Ecological Methodology 2a ed. University of British Columbia, USA. 620 pp.

Krebs, J. R. y N. B. Davies. 2009. Behavioural ecology: an evolutionary approach. John Wiley \& Sons, 500 p.

Langton, R. W. 1982. Diet overlap between Atlantic cod, Gadus morhua, silver hake, Merluccius bilinearis, and fifteen other northwest Atlantic finfish. Fishery Bulletin, 80: 745-759.

Levins, R. 1968. Evolution in changing environments: some theoretical explorations (No. 2). Princeton University Press, NJ, USA, 120 pp.

Lewis J. B. 1964. Feeding and digestion in the tropical sea urchin Diadema antillarum Philippi Canadian Journal of Zoology, 42: 550-557.

MacArthur, R. H. y E. R. Pianka. 1966. On optimal use of patchy environment. American Naturalist, 100: 603-609. 
Magurran, A. E. 2004. Measuring Biological Diversity. Blackwell Science, Oxford, 256 pp.

McClanahan T. R. 1988. Coexistence in a sea urchin guild and its implications to coral reef diversity and degradation. Oecologia, 77: 210-218.

McClanahan, T. R. y N. A. Muthiga. 2013. Ecology of Echinometra, p. 337-353. En: Edible Sea Urchins: Biology and Ecology, JM Lawrence (Ed.), Elsevier Science, 432 pp.

Martínez, D., N. Romero y D. Matos. 2016. Diversidad de equinodermos en las pocetas intermareales de Guaimaral y La Sardina. Informe Científico, Departamento de Biología, Universidad de Oriente, Cuba, 21 pp.

McClintock, J. B., T. S. Klinger y J. M. Lawrence. 1982. Feeding preferences of echinoids for plant and animal food models. Bulletin of Marine Science, 32: 365-369.

Monroy, M. y O. D. Solano. 2005. Estado poblacional de Echinometra lucunter (Echinoida: Echinometridae) y su fauna acompañante en el litoral rocoso del Caribe Colombiano. Revista de Biología Tropical, 53 (3): 291-297.

Montealegre-Quijano, S. y A. Gómez Gaspar. 2005. Ciclo reproductivo de Lytechinus variegatus (Echinoidea: Toxopneustidae) en el sur de Isla Margarita, Venezuela. Revista de Biología Tropical, 53 (3): 305-312.

Muthiga, N. A. y T. R. McClanahan. 2007. Ecology of Diadema, p. 205-225. En: Edible Sea Urchins: Biology and Ecology, JM Lawrence (Ed.), Elsevier Science, 529 pp.

Nappi, A. y J. Crawford. 1984. The ocurrence and distribution of a syndesmid (Turbellaria: Umagillidae) in Jamaican sea urchins. Journal Parasitology, 7: 595-597.

Pimm S. L. y J. H. Lawton. 1978. On feeding on more than one trophic level. Nature, 275: $542-544$.

Pinnegar, J. K. y N. V. C. Polunin. 2000. Contributions of stable-isotope data to elucidating food webs of Mediterranean rocky littoral fishes. Oecologia, 122 (3): 399-409.

Reyes-Luján, J., J. Barrios, D. Arriche, E. Zapata-Vivenes, W. Salgado y C. Lodeiros. 2015. Dieta del erizo negro Echinometra lucunter (Echinometra: Echinoidea) en el Nororiente de Venezuela. Revista de Biología Tropical, 63: 233-242.

Rivas, L. R. 1964. A reinterpretation of the concepts "Sympatric" and "Allopatric" with proposal of the additional terms "Syntopic" and "Allotopic". Systematic Zoology, 13: 42-43.

Rodríguez-Barreras, R., E. Cuevas, N. Cabanillas-Teran y A. Sabat. 2015. Potential omnivory in the sea urchin Diadema antillarum? Regional Studies in Marine Science, 2: 11-18.

Rodríguez-Barreras, R., E. Cuevas, N. Cabanillas-Terán y B. Branoff. 2016. Understanding trophic relationships among Caribbean Sea urchins. Revista de Biología Tropical, 64 (2): 837-848.

Sala, E., C. F. Boudouresque y M. Harmelin-Vivien. 1998. Fishing, trophic cascades, and the structure of algal assemblages: evaluation of an old but untested paradigm. Oikos, 82 (3): 425-439. 
Shulman, M. J. 1990. Aggression among sea urchins on Caribbean coral reefs. Journal of Experimental Marine Biology and Ecology, 17: 181-186.

Svärdson, G. 1949. Competition and habitats selection in birds. Oikos, 1: 157-174.

Taper, M. L. y P. A. Marquet. 1996. How do species really divide resources? The American Naturalist, 147: 1072-1082.

Vanderklift, M. A., G. A. Kendrick, y A. J. Smith. 2006. Differences in trophic position among sympatric sea urchin species. Estuarine, Coastal and Shelf Science, 66: 291-297.

Watts, S., J. McClintocka y J. Lawrence. 2007. Ecology of Lytechinus, p. 473-497. In: Edible Sea Urchins: Biology and Ecology, JM Lawrence (Ed.), Elsevier Science, 529 pp.

Whittaker, R. H. 1965. Dominance and diversity in land plant communities. Science, 147 (3655): 250-260.

Williams, A. H. 1980. The three-spot damshelfish: a noncarnivorous keystone species. The American Naturalist, 116: 138-142.

Wing, S. R., R. J. McLeod, K. L. Clark y R. D. Frew. 2008. Plasticity in the diet of two echinoderm species across an ecotone: microbial recycling of forest litter and bottom-up forcing of populations structure. Marine Pollution Bulletin, 360: 115-123. 